\title{
Environmental Economic Dispatch Optimization using a Modified Genetic Algorithm
}

\author{
Simona Dinu \\ Sr. Lecturer \\ Constanta Maritime University \\ Mircea cel Batran Constanta \\ ROMANIA
}

\author{
loan Odagescu \\ Professor \\ The Bucharest Academy of \\ Economic Studies \\ ROMANIA
}

\author{
Maria Moise \\ Professor \\ Romanian American \\ University, Bucharest \\ ROMANIA
}

\begin{abstract}
This paper aims to develop a new Genetic Algorithm based approach to solve the Combined Environmental Economic Power Dispatch Problem. The essential features of our proposed algorithm include a diploid based complex-encoding with meiosis specific attributes and new mutation operators that performs global search during the initial generations and local search in the later generations. Using the parallel searching mechanism and the new defined mutation operators, the local searching ability of the algorithm is improved, as well as the algorithm's efficiency.
\end{abstract}

Results of comparative tests on a sample power system are presented, showing the better computation efficiency and convergence property of the proposed methodology.

\section{General Terms}

Dispatch strategies. Evolutionary Computation. Energy Systems.

\section{Keywords}

Multiobjective optimization. Environmental Economic Dispatch, Genetic Algorithms. Diploidy.

\section{INTRODUCTION}

The dispatch strategy for an energy system is a primary objective in the operation of power systems. It is a computational process based on a set of rules controlling the interaction among various system components in order to operate the power system in an economic and efficient manner.

In accordance with the objectives, the dispatch strategies can be divided in:

- economic dispatch, attempting to schedule the committed generating unit outputs to meet the load demand at minimum production and transmission (operating) cost;

- environmental dispatch, attempting to reduce the environmental impact of power generation ;

- economic/environmental dispatch, attempting to achieve both objectives (minimizing the operating fuel cost and emission cost) in a single dispatch.

The classical Economic Dispatch (ED) is to allocate the total required generation among the available generating units in order to minimize the total generation cost while simultaneously satisfying all equality and inequality constraints. To balance the load variations, the power output of generators has to be adapted. This leads to minimizing system losses at all time and decrease the operational costs. Thus, it is a critical task of electric utilities to deliver power as demanded in order to maintain the reliability and continuity of electricity supply. The literature of the ED problems and its different numerical solution methods are investigated in [1] [2].

Fossil-fueled electric power plants produce harmful emission such as Sulfur Oxides, Nitrogen Oxides and Carbon Dioxide. Recently, in order to meet severe environmental standards imposed by legislation, pollution minimization has become another important operational goal. Thus, improvements in scheduling the unit outputs must result in both monetary profits and reduced emissions of gaseous pollution. A survey of the commonly environmental dispatch algorithms has been given in [3] and [1].

The Combined Environmental Economic Dispatch (CEED) problem is a bicriterial optimization problem with two conflicting objective functions: operating costs and environmental impact of emissions. Due to the contrasting/conflicting goals and non-commensurable natures of fuel cost and emission minimization objectives, conventional approach which optimizes the integrated two objective functions seems not appropriate for this class of multiobjective optimization problems [4].

Therefore, conventional optimization methods based on derivatives and gradients are not suitable for this nonlinear and multimodal optimization problem.

Not longer after considering the environmental feature in the ED problem, different solution methods have progressively been reported in the literature concerning the CEED problem. Researchers' methods considered emissions either in the objective function (by converting into a single-objective problem and assigning relative weights to each objective [5], [6]) or treated them as additional operational constraints that must be satisfied [7], [8].

With increasing size and complexity of the problem, many researchers have proposed the use of heuristic optimization approaches. Evolutionary computation techniques such as genetic algorithms (GA), evolutionary strategies (ES), evolutionary programming (EP), genetic programming (GP) and related fields such as swarm intelligence (Ant Colony Optimization and Particle Swarm Optimization) and other 
evolutionary computation techniques are suited to deal with the problem at hand.

\section{THE PROBLEM FORMULATION}

Input-output characteristics of power generation units are the most important initial data for solving the problem of optimal planning and operation of power plants.

The input for a thermal plant (fuel input) is usually measured in $\mathrm{MJ} / \mathrm{h}$ (or Btu/h), and the output (power output) is measured in MW. The input for a hydroelectric unit is expressed in terms of volume of water per unit time $(\mathrm{m} 3 / \mathrm{h})$ and the output is the same (electric power)

The widely used input-output characteristic of the ith generating unit is a quadratic function [9]:

$$
H T\left(P_{G i}\right)=a_{i} P_{G i}^{2}+b_{i} P_{G i}+c_{i} \ldots . . B t u / h
$$

where the suffix i stands for the unit number.

HT is the heat input, PGi is the net output power and ai , bi, and ci are the coefficients of the input - output characteristic.

The constant ci is equivalent to the fuel consumption of the generating unit operation without power output.

A typical input-output curve is shown in Figure 1:

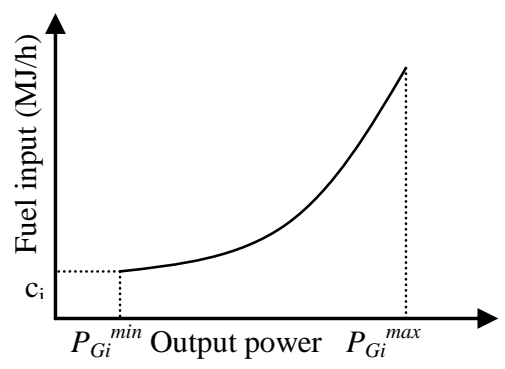

Figure 1. Input-output curve

The $P_{G i}{ }^{\min }$ and $P_{G i}{ }^{\max }$ are the minimum and maximum limit on the generated output of the $\mathrm{i}^{\text {th }}$ generating unit:

$$
P_{G i}^{\min } \leq P_{G i} \leq P_{G i}^{\max }
$$

Such of curves are developed for each generating unit involved. The fuel cost characteristics (FCC) is calculated by multiplying the fuel input with the corresponding fuel cost ( $\mathrm{K}=$ constant) expressed in an arbitrary monetary unit (UM):

$$
\operatorname{FCC}\left(P_{G i}\right)=K^{*} H T\left(P_{G i}\right)
$$

Thus, the FCC for the unit i can be written in the form of a quadratic polynomial similar to the heat input equation:

$$
\operatorname{FCC}\left(P_{G i}\right)=a_{i} P_{G i}^{2}+b_{i} P_{G i}+c_{i} \ldots \ldots . U M
$$

\subsection{Objective functions}

\section{Fuel cost objective}

If there are $\mathrm{n}$ generators committed to the system and the active power load PD is given, then the ED problem can therefore be stated as:

Minimize $F C C\left(P_{G}\right)=\sum_{i=1}^{n} F C C\left(P_{G_{i}}\right)$,

the total generation cost subject to given constraints:

(i) $\sum_{i=1}^{n}\left(P_{G_{i}}\right)=P_{D}+P_{\text {Loss }}$

equality constraint: the total system generation equals the total system load $\left(\mathrm{P}_{\mathrm{D}}\right)$ plus system losses $\left(\mathrm{P}_{\text {Loss }}\right)$.

(ii) $P_{G i}^{\min } \leq P_{G i} \leq P_{G i}^{\max }, i=1, \ldots, n$

the inequality constraints: active power output of each generating unit is ranging between its lower and upper limits to ensure stable operation.

$\mathrm{P}_{\mathrm{G}}$ is the vector of real power outputs of generators and is defined as $\mathrm{P}_{\mathrm{G}}=\left[\mathrm{P}_{\mathrm{G} 1}, \mathrm{P}_{\mathrm{G} 2}, \ldots, \mathrm{P}_{\mathrm{Gn}}\right]^{\mathrm{T}}$.

Using the B-coefficient method, network losses are expressed using George's formula [10]:

$$
P_{\text {Loss }}=\sum_{i=1}^{n} \sum_{j=1}^{n} P_{G_{i}} B_{i j} P_{G_{j}}
$$

where $\mathrm{Bij}=$ loss (or B-coefficients).

Further constraints can be added depending on the study requirements: valve point effects, the use of multiple fuel types, prohibited operating zones, etc.

\section{Emission objective}

The amount of emission from an unit (such as $\mathrm{N}_{\mathrm{ox}}, \mathrm{S}_{\mathrm{ox}}, \mathrm{C}_{\mathrm{ox}}$, thermal emissions, etc.) depends on the amount of power generated by the unit. Thus, in order to solve a CEED model, emissions must be expressed by functions that relate emissions with power production for each unit. Generally, the total ton/h emission of these pollutants can be expressed as:

$$
E\left(P_{G}\right)=\sum_{i=1}^{n}\left(\alpha_{i} P_{G i}^{2}+\beta_{i} P_{G i}+\gamma_{i}\right) \ldots \ldots . K g / h
$$

Also, $\alpha_{\mathrm{i}}, \beta_{\mathrm{i}}$ and $\gamma_{\mathrm{i}}$ are the emission coefficients of generator i. The pollution control cost can be obtained by assigning a cost factor to the pollution level expressed as:

$$
\operatorname{PCC}\left(P_{G}\right)=W^{*} E\left(P_{G}\right)
$$




\subsection{Multiobjective Economic/Environmental dispatch formulation}

A multiobjective optimization problem can be stated as follows:

$$
\begin{aligned}
\text { opt } F(x)=( & \left.f_{1}(x), \ldots, f_{p}(x)\right) \\
\text { subject to: } & \left\{\begin{array}{l}
x \in \Omega \\
g_{i}(x) \geq 0 \\
h_{j}(x) \leq 0
\end{array}\right.
\end{aligned}
$$

where:

$\Omega$ is the decision space (the finite set of feasible solutions)

$\mathrm{R}^{\mathrm{p}}$ is the objective space

$\mathrm{F}: \Omega \rightarrow \mathrm{R}^{\mathrm{p}}$ consists of $\mathrm{p}$ real valued objective functions $\mathrm{g}_{\mathrm{i}}(\mathrm{x})$ and $\mathrm{h}_{\mathrm{j}}(\mathrm{x})$ are the nonlinear and linear constraints.

The solutions that are non-dominated within the entire search space are denoted as Pareto-optimal solutions and represent optimal trade-offs among all objectives. A Pareto optimal solution cannot be improved with respect to any objective without worsening at least one other objective.

The combined environmental and economic dispatch (CEED) problem can be formulated and solved as a multiobjective optimization problem:

$$
\text { Minimize }\left[F C C\left(P_{G}\right), P C C\left(P_{G}\right)\right]
$$

\section{CLASSICAL LAMBDA ITERATION METHOD FOR THE CEED PROBLEM}

The total objective function combines the two objectives (cost of generation and cost of the pollution level control) into a single objective function by using a price penalty factor $h$ :

$$
F_{T}=F C C\left(P_{G}\right)+h * P C C\left(P_{G}\right)
$$

There are various price penalty factors suggested in the literature [8]. In this article the maximum price penalty factor for a generator is considered:

$$
h=\frac{F C C\left(P_{G i}^{\max }\right) / P_{G i}^{\max }}{P C C\left(P_{G j}^{\max }\right) / P_{G j}^{\max }}
$$

Where: $i$ is the highest fuel-cost unit $\mathrm{j}$ is the highest pollutant-emission unit

Using Lagrangian Multipliers, an augmented function for the CEED problem can be written as:

$$
L\left(P_{G}, \lambda\right)=F_{T}+\lambda\left(P_{D}+P_{\text {Loss }}-\sum_{i=1}^{n}\left(P_{G i}\right)\right)
$$

Necessary conditions for the optimization are:

$$
\begin{aligned}
& \text { (i) } d L\left(P_{G i}, \lambda\right) / d P_{G i}=0-\text { coordination equation } \\
& \text { (ii) } d L\left(P_{G i}, \lambda\right) / d \lambda=0
\end{aligned}
$$

Rearranging the coordination equation,

$$
\begin{aligned}
& 2\left(a_{i}+h_{i} \alpha_{i}\right) P_{G i}+\left(b_{i}+\beta_{i} h_{i}\right)= \\
& \lambda\left(1-d P_{L o s s} / d P_{G i}\right) \\
& \text { where } d P_{L o s s} / d P_{G i}=\sum_{j=1}^{n} 2 B_{i j} P_{G j}
\end{aligned}
$$

The initial value chosen for lambda is the mid-point of the interval $\left(\lambda_{\min }, \lambda_{\max }\right)$, i.e $\lambda=\left(\lambda_{\min }+\lambda_{\max }\right) / 2$, by assuming that the transmission losses are zero:

$$
\begin{aligned}
& \lambda_{\text {min }}=\min _{i=1, \ldots, n}\left\{d F_{T}\left(P_{G i}^{\min }\right) / d P_{G i}\right\} \\
& \lambda_{\text {max }}=\max _{i=1, \ldots, n}\left\{d F_{T}\left(P_{G i}^{\max }\right) / d P_{G i}\right\}
\end{aligned}
$$

Then the coordination equation is solved iteratively (imposing the generator power limits) until equation (6) is satisfied by modifying $\lambda$. This technique is known as successive approximation.

\section{THE PROPOSED APPROACH}

In this study, a random-weighted GA (RWGA) [11] based on complex-encoding is proposed. A normalized vector $w^{i}=\left(w_{1}^{i}, w_{2}^{i}\right)$ is randomly generated for each solution $x_{i} \in P^{(t)}$ during the selection phase at each generation. By changing weights during the running time, this approach provides multiple search directions and thus an increased ability to evaluate the area uniformly over the entire frontier.

The total objective function combines the two objectives (cost of generation and cost of the pollution level control) into a single objective function by using a weighted sum:

$$
F_{T}(x)=w_{1} F C C(x)+h w_{2} P C C(x)
$$

The problem is subject to the equality constraint given in equation (6) and generation limits inequality constraint given in equation (7).

This procedure applied in our study is given as follows:

Step1(Initialization): The GA begins with generating a random initial population $\mathrm{P}^{(0)}$ of candidate solutions.

Set $\mathrm{t}=0$.

Step2(Evaluation): Assign a fitness value to each solution $\mathrm{x}_{\mathrm{i}} \in$ $\mathrm{P}^{(\mathrm{t})}$ by performing the follow steps:

(i) Generate the weights as follows:

$$
\begin{aligned}
& w_{1}(t)=\frac{1}{2}\left|\sin \left(\frac{2 \pi t}{G_{\max }}\right)+\cos \left(\frac{2 \pi t}{G_{\max }}\right)\right| \\
& w_{2}(t)=1-w_{1}(t)
\end{aligned}
$$

where $\mathrm{t}=$ number of generation, $\mathrm{t}=1,2, \ldots, \mathrm{G}_{\max }$ $\mathrm{G}_{\max }$ is the maximum number of generations.

(ii) Calculate: objective function from equation (20) 
Step 3(Selection): Minimizing $\mathrm{F}$ is based on finding a maximum fitness value in the searching process:

$$
\begin{aligned}
& f_{i}=\operatorname{fitness}\left(x_{i}\right)=\frac{f^{\max }\left(x_{i}\right)}{F\left(x_{i}\right)}, \\
& f^{\max }\left(x_{i}\right)=\left\{\max F\left(x_{i}\right) \mid x_{i} \in P^{(t)}\right\}
\end{aligned}
$$

Calculate the selection probability of each solution $\mathrm{x}_{\mathrm{i}} \in \mathrm{P}^{(\mathrm{t})}$ :

$$
p_{i}=\frac{f_{i}-f^{\mathrm{min}}}{\sum_{j=1}^{P S}\left(f_{j}-f^{\mathrm{min}}\right)}
$$

Where: PS is the population size;

$$
f^{\min }=\left\{\min F(x) \mid x \in P^{(t)}\right\}
$$

Select parents using the fitness-proportion selection based on probabilities calculated in (23).

Step 4(Genetic operators): Thereafter crossover and mutation operators are applied on the population.

Step 5: If the stopping condition is satisfied, return $\mathrm{P}^{(\mathrm{t})}$. Otherwise set $\mathrm{t}=\mathrm{t}+1$ and go to Step 2 .

\subsection{Mapping of the problem}

Chromosome representation is an essential component of any genetic algorithm. One of the major advantages that increase GA's accuracy and ability in searching for feasible solutions is the flexibility of chromosome representation.

The most common representation of genetic information in GA is based on the haploid model, i.e. one string of genetic information for each individual in the population; the sting can be represented by binary code, gray code or real code.

The concept of diploidy in human genetics has inspired researchers in Evolutionary Computation to develop new diploid encoding schemes [12], [13].

Goldberg and Smith [14] reported the first experimental and theoretical results. Since then, various studies have focused on the effects of the cardinality of genotypic representation. A comparison of the results obtained in the literature [15] demonstrated that diploidy-based GA retains greater diversity and shows more robustness than simple GA.

In this study we use complex-encoding GA based on diploid genotype [16]. To improve its performance, we propose meiosis specific features: duplication and recombination with real valued representation scheme for solution (Figure 2) and a new mutation operator for imaginary genes of the chromosomes.

A population of constant size PS consisting of n-dimensional real-valued diploid chromosomes is given by:

$$
P^{(t)}=\left[C_{1}^{(t)}, \ldots, C_{P S}^{(t)}\right], t=1, \ldots, G_{\max }
$$

An individual (chromosome) is a n-dimension vector given by:

$$
C_{k}^{(t)}=\left(P_{k 1}^{(t)}, \ldots, P_{k n}^{(t)}\right), k=1, \ldots, P S
$$

and each gene $P_{k i}^{(t)}$ is a pair $\left(\rho_{k i}^{(t)}, \theta_{k i}^{(t)}\right)$ consisting of the modulus and angle of the complex representation of that allele.

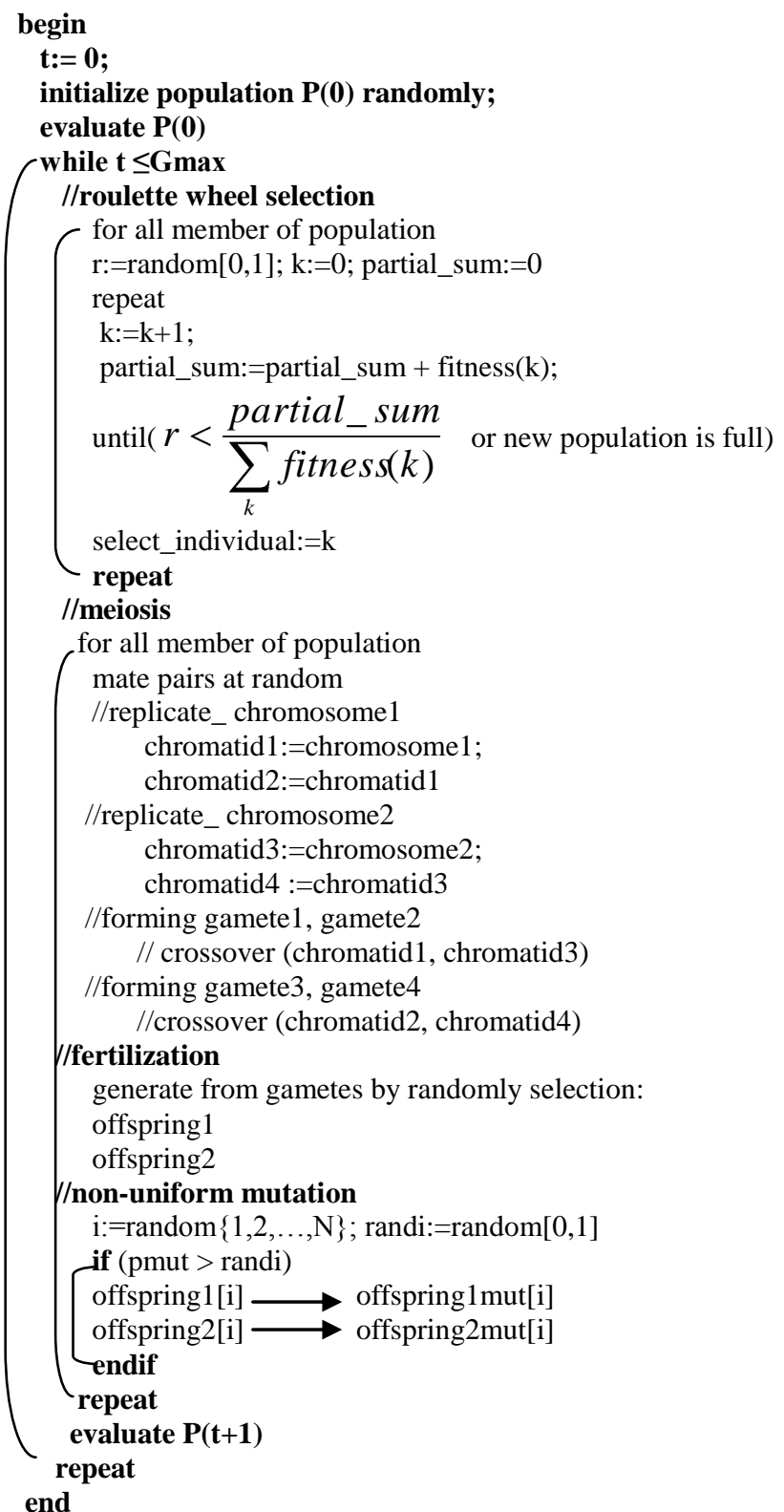

\section{Figure 2. The proposed GA for CEED problem}

Initialization:

The initial population is randomly generated using equations (27) and (28) as follows: 


$$
\begin{aligned}
\rho_{k i}^{(1)} & =[\text { rand }] \times \frac{P_{G i}^{\max }-P_{G i}^{\min }}{2} \\
\theta_{k i}^{(1)} & =[\text { rand }] \times 2 \pi, k=1, \ldots, P S ; i=1, \ldots, n
\end{aligned}
$$

The resultant gene $P_{k i}^{(1)}$ which corresponds to an allele is given by:

$P_{k i}^{(1)}=\rho_{k i}^{(1)} \times \cos \theta_{k i}^{(1)}+\frac{P_{G i}^{\max }+P_{G i}^{\min }}{2}$

$k=1, \ldots, P S ; i=1, \ldots, n$

One can note that:

$P_{G i}^{\min } \leq P_{k i}^{(1)} \leq P_{G i}^{\max }, k=1, \ldots, P S ; i=1, \ldots, n$

\section{Evaluation and selection:}

Once the individuals of current population are evaluated according to their fitness, the individuals that will be the parents of the next generation are selected according to the desired selection scheme. This study uses the proportional (roulette wheel) selection. Next, the selected individuals are paired off randomly to give rise to new offsprings.

\section{Genetic operators:}

The reproduction of the individuals in this study is inspired by the organic mechanism of a meiotic cell division. In this context, the term "meiosis" refers to the process whereby a nucleus divides by two divisions (meiosis I and meiosis II) into four gametes. Meiosis halves the number of chromosomes before sexual reproduction, thereby ensuring that chromosome number does not double with each generation. Before meiosis, each chromosome is replicated, forming two sisters "chromatids" that remain linked together.

The two sister chromatids forming each homolog are then separated during the second meiotic division.

In order to perform crossover for two chosen chromatids, both the modules and angles of the arguments are changed as described below.

Let us consider that we perform the crossover operator for chromatid 1 and chromatid 3 .

Their modules are:

$\left\{\begin{array}{l}\rho_{1}=\left(\rho_{11} \ldots \rho_{k 1} \ldots \rho_{n 1}\right) \text { and respectively } \\ \rho_{3}=\left(\rho_{13} \ldots \rho_{k 3} \ldots \rho_{n 3}\right)\end{array}\right.$

and their angles are:

$\left\{\begin{array}{l}\theta_{1}=\left(\theta_{11} \ldots \theta_{k 1} \ldots \theta_{n 1}\right) \text { and respectively } \\ \theta_{3}=\left(\theta_{13} \ldots \theta_{k 3} \ldots \theta_{n 3}\right)\end{array}\right.$

After performing their crossover, two gametes are obtained: gamete $_{1}$ has:

$$
\left\{\begin{array}{l}
\rho_{c_{1}}=\left(\ldots, r \times \rho_{k 1}+(1-r) \times \rho_{k 3}, \ldots . .\right) \\
\theta_{c_{1}}=\left(\ldots, r \times \theta_{k 1}+(1-r) \times \theta_{k 3}, \ldots .\right)
\end{array}\right.
$$

gamete $_{2}$ has:

$$
\left\{\begin{array}{l}
\rho_{c_{2}}=\left(\ldots,(1-r) \times \rho_{k 1}+r \times \rho_{k 3}, \ldots .\right) \\
\theta_{c_{2}}=\left(\ldots .,(1-r) \times \theta_{k 1}+r \times \theta_{k 3}, \ldots .\right)
\end{array}\right.
$$

where $\mathrm{r}$ is a random number between 0 and 1 .

The probability of crossover is $p_{c}$, so that an average of $p_{c} x$ $100 \%$ chromosomes undergo crossover.

Fertilization (putting together two gametes resulted from meiosis) is done by randomly combining gametes from the gene pool: two of the gametes from the four that have been formed, are then selected randomly to form two new offsprings.

The next genetic operator, mutation, is a mechanism for extending the search on the new areas of search space. Mutation modifies the genotype, and thus the phenotype, by random altering of bit's values inside chromosome with given probability. In this paper we use non-uniform mutation [9] for the module of an argument.

Let us consider that we perform the non-uniform mutation operator for a resultant offspring.

If $\rho=\left(\rho_{1} \ldots \rho_{k} \ldots \rho_{n}\right)$ is the modulus component and $\rho_{k}$ is selected at random for mutation, the result is:

$$
\rho^{\text {mut }}=\left(\rho_{1} \ldots \rho_{k}^{\text {mut }} \ldots \rho_{n}\right) \text {, where: }
$$

$\rho_{k}^{m u t}=\left\{\begin{array}{l}\rho_{k}+\left(P_{G i}^{\max }-\rho_{k}\right) f(\text { gen }), r_{1}<0.5 \\ \rho_{k}+\left(\rho_{k}-P_{G i}^{\mathrm{min}}\right) f(\text { gen }), r_{1}>0.5 \\ \rho_{k}, \text { otherwise }\end{array}\right.$

Where $f(g e n)=\left(r_{2}\left(1-\frac{g e n}{G_{\max }}\right)\right)^{\tau}$

$\mathrm{r}_{1}, \mathrm{r}_{2}$ are randomly generated numbers in interval $(0,1)$; gen is the current generation;

$\tau$ is a system parameter determining the degree of dependency on the iteration number. In this study we set $\tau=3$.

For an angle of an argument we propose a new mutation operator defined as follows:

if $\theta=\left(\theta_{1} \ldots \theta_{k} \ldots \theta_{n}\right)$ is the angle component and $\theta_{k}$ is selected at random for mutation, the result is: $\theta^{m u t}=\left(\theta_{1} \ldots \theta_{k}^{m u t} \ldots \theta_{n}\right)$, where:

$\theta_{k}^{\text {mut }}=\left\{\begin{array}{l}\max \left(\theta_{k}-f(\text { gen }) \cdot \pi, 0\right), r_{1}<0.5 \\ \min \left(\theta_{k}+f(\text { gen }) \cdot \pi, 2 \pi\right), r_{1}>0.5 \\ \theta_{k}, \text { otherwise }\end{array}\right.$

Where $f(g e n)=1-r_{2}^{\left(1-\frac{g e n}{G_{\max }}\right)^{\tau}}$

and the other parameters are the same as those described above. As can be seen from equations (33) and (35), the amplitude of the change decrease as one approaches the maximum number of generations. Thus, these mutation operators perform global search during the initial generations and local search in the later 
generations. Moreover, the local searching ability of the algorithm is improved, as well as the algorithm's efficiency.

A large number of studies which explore the interactions among different GA parameters showed that in general GAs will work well with high crossover and low mutation probability. Therefore, common to each run were the following parameter settings: population size was 50 , number of generations was 1000 , crossover rate was $80 \%$ and mutation rate was $3 \%$.

\subsection{APPLICATION STUDY}

The techniques used in this study and simulations were carried out using $\mathrm{C}++$ language in the Microsoft Visual Studio Development environment. The algorithm gives:

- Best solutions with respect to individual objectives: fuel cost and emission objectives are optimized individually;

- Best compromise solution obtained by performing multiobjective optimization.

Studies were performed on a CEED problem as in [17], [18] for a six-generator system. The generator cost coefficients, emission coefficients and generation limits of units system are taken from the ones above and are given in Table 1 and in Table 2.

For comparison purposes with the reported results, the system is considered as lossless, although the proposed algorithm permits their consideration.

Table 1. Fuel cost and emission coefficients

\begin{tabular}{|l|l|l|l|l|l|l|}
\hline \multirow{2}{*}{ Unit } & \multicolumn{4}{|l|}{ Cost function } & \multicolumn{3}{l|}{ Emissions cost } \\
\cline { 2 - 7 } & $\mathbf{a}_{\mathbf{i}}$ & $\mathbf{b}_{\mathbf{i}}$ & $\mathbf{c}_{\mathbf{i}}$ & $\boldsymbol{\alpha}_{\mathbf{i}}$ & $\boldsymbol{\beta}_{\mathbf{i}}$ & $\gamma_{\mathbf{i}}$ \\
\hline 1 & 0.15247 & 38.53973 & 756.79886 & 0.00419 & 0.32767 & 13.85932 \\
\hline 2 & 0.10587 & 46.15916 & 451.32513 & 0.00419 & 0.32767 & 13.85932 \\
\hline 3 & 0.02803 & 40.39655 & 1049.9977 & 0.00683 & -0.54551 & 40.2669 \\
\hline 4 & 0.03546 & 38.30553 & 1243.5311 & 0.00683 & -0.54551 & 40.2669 \\
\hline 5 & 0.02111 & 36.32782 & 1658.5696 & 0.00461 & -0.51116 & 42.89553 \\
\hline 6 & 0.01799 & 38.27041 & 1356.6592 & 0.00461 & -0.51116 & 42.89553 \\
\hline
\end{tabular}

Table 2. Operating limits

\begin{tabular}{|l|l|l|}
\hline Generator & $\begin{array}{l}\text { Lower limit } \\
\left(\boldsymbol{P}_{G i}^{\text {min }}\right)\end{array}$ & $\begin{array}{l}\text { Upper limit } \\
\left(\boldsymbol{P}_{G i}^{\text {max }}\right)\end{array}$ \\
\hline 1 & 10 & 125 \\
\hline 2 & 10 & 150 \\
\hline 3 & 35 & 225 \\
\hline 4 & 35 & 210 \\
\hline 5 & 130 & 325 \\
\hline 6 & 125 & 315 \\
\hline
\end{tabular}

A total load of $500 \mathrm{MW}$ is considered and fuel cost and emission objectives are at first optimized individually in order to explore the extreme points obtained by the proposed approach:

- Pure Economic Objective: $\mathrm{w}_{1}=1, \mathrm{w}_{2}=0$

- Pure Emission Objective: $\mathrm{w}_{1}=0, \mathrm{w}_{2}=1$

The best results of cost and emission when optimized individually and the best compromise solution are given in Table 3. Convergence characteristics of best fuel cost and best emission are shown in Figure 3.

These graphs clearly indicate that the proposed approach converges rapidly to the optimal solution.

Table 3. Best individually optimized objectives and best compromise

\begin{tabular}{|l|l|l|l|}
\hline Generator & Best fuel cost & Best emission & Best compromise solution \\
\hline $\mathrm{P}_{\mathrm{G} 1}$ & 23.892 & 49.186 & 25.731 \\
\hline $\mathrm{P}_{\mathrm{G} 2}$ & 21.243 & 44.681 & 22.149 \\
\hline $\mathrm{P}_{\mathrm{G} 3}$ & 88.741 & 63.169 & 89.154 \\
\hline $\mathrm{P}_{\mathrm{G} 4}$ & 74.143 & 72.119 & 92.152 \\
\hline $\mathrm{P}_{\mathrm{G} 5}$ & 150.792 & 131.172 & 141.124 \\
\hline $\mathrm{P}_{\mathrm{G} 6}$ & 141.189 & 139.673 & 129.690 \\
\hline Fuel cost & $\mathbf{2 6 9 1 4}$ & $\mathbf{2 8 1 2 0}$ & $\mathbf{2 7 0 8 9 . 7 9}$ \\
\hline Emission & $\mathbf{2 6 8 . 4 9}$ & $\mathbf{2 3 9 . 1 2}$ & $\mathbf{2 6 1 . 2 1 9}$ \\
\hline
\end{tabular}

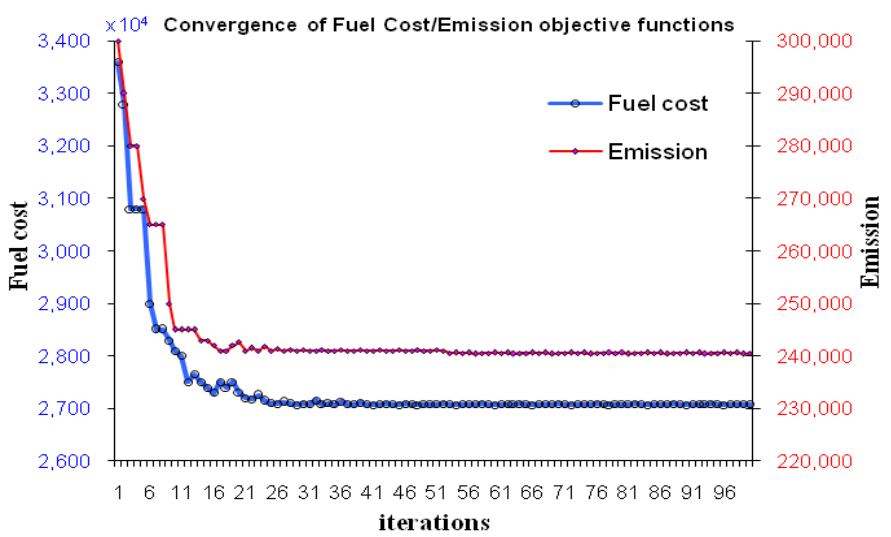

Figure 3. Convergence of fuel cost and emission objective functions 
The optimum solution for combined environmental and economic dispatch is given in Table 5. The results are compared against the results obtained from conventional $\lambda$-iteration method [19] and Recursive method [20].The execution time of the proposed algorithm for this 6-unit system was about 0.3 seconds for all cases (individually optimization/combined environmental and economic optimization). From the results, it is quite evident that the proposed approach outperforms the other two methods. This demonstrates the performance and applicability of the proposed approach in solving this multiobjective optimization problem.

Tables 3 and 5 also reveal that the total fuel cost and total emission are in between the respective values of separate economic and emission optimization. Figure 4 shows the best objective values obtained by the 100 runs performed when using the proposed algorithm. The average and standard deviation for these 100 runs are shown in the figure.
Table 4 includes the average, maximum, minimum and standard deviation obtained after performing these independent runs. "Hits" is the number of runs in which we obtained a solution differing by less than $0,1 \%$ from the best solution obtained. "Average" means the best objective function value for 100 runs of the algorithm. It shows the quality of candidate solutions through iterations. The difference between $\max$ and $\min$ objective values expresses the search range of the algorithms. "St. Dev" denotes standard deviation, which expresses the searching capacity of each algorithm. As Table 4, the proposed algorithm has a small mean value and a small standard deviation. That is, this algorithm has better convergence stability, is more robust and is able to reach good solutions across 100 runs.

Table 4. Performances of the proposed algorithm

\begin{tabular}{|l|l|l|l|}
\hline 100 runs & Hits & Average & St.dev \\
\hline$[27086.07,27094.96]$ & 17 & 27089.79 & 3.24 \\
\hline
\end{tabular}

Table 5. Comparison of test results obtained from the above mentioned methods

\begin{tabular}{|l|l|l|l|l|l|l|l|l|}
\hline Method & $\begin{array}{l}\text { P1 } \\
\text { (MW) }\end{array}$ & $\begin{array}{l}\text { P2 } \\
\text { (MW) }\end{array}$ & $\begin{array}{l}\text { P3 } \\
\text { (MW) }\end{array}$ & $\begin{array}{l}\text { P4 } \\
\text { (MW) }\end{array}$ & $\begin{array}{l}\text { P5 } \\
\text { (MW) }\end{array}$ & $\begin{array}{l}\text { P6 } \\
\text { (MW) }\end{array}$ & $\begin{array}{l}\text { Fuel } \\
\text { (\$/h) })\end{array}$ & $\begin{array}{l}\text { Emission } \\
(\mathbf{k g} / \mathbf{h})\end{array}$ \\
\hline $\begin{array}{l}\text { Proposed } \\
\text { method }\end{array}$ & 25.731 & 22.149 & 89.154 & 92.152 & 141.124 & 129.690 & $\mathbf{2 7 0 8 9 . 7 9}$ & $\mathbf{2 6 1 . 4 1 9}$ \\
\hline $\begin{array}{l}\text { Conv. } \\
\lambda \text {-iteration }\end{array}$ & 21.119 & 22.047 & 79.214 & 99.611 & 149.418 & 128.591 & $\mathbf{2 7 0 9 2 . 5 0}$ & $\mathbf{2 6 1 . 6 3 5}$ \\
\hline $\begin{array}{l}\text { Recursive } \\
\text { method }\end{array}$ & 26.124 & 28.246 & 68.421 & 97.125 & 147.115 & 132.969 & $\mathbf{2 7 0 9 2 . 5 0}$ & $\mathbf{2 6 1 . 6 3 4}$ \\
\hline
\end{tabular}

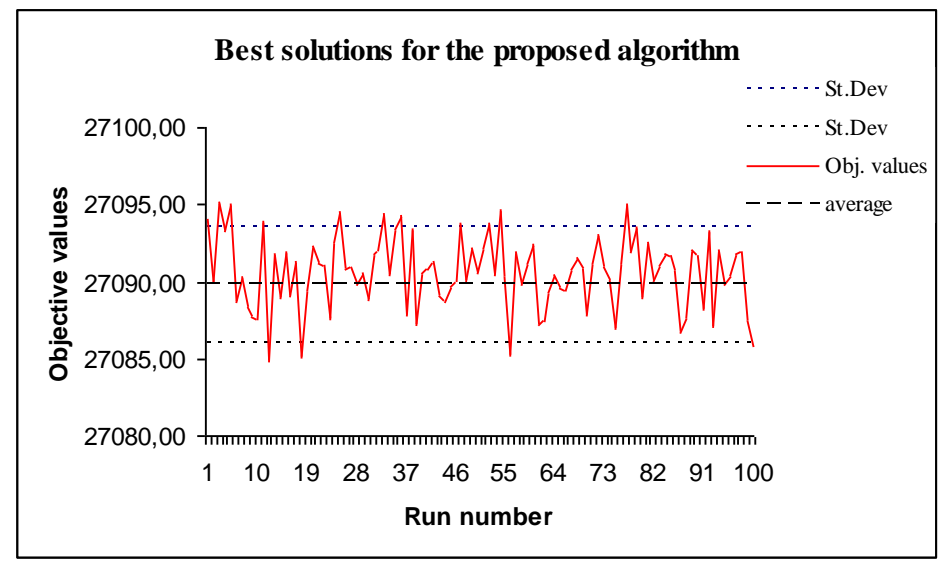

Figure 4. Best objective values for the proposed algorithm - 100 runs

\section{CONCLUSIONS}

A novel form of Genetic Algorithm is presented in this study. The essential features of our proposed algorithm include a diploid based complex-encoding with meiosis specific features and new mutation operators that performs global search during the initial generations and local search in the later generations. Using the parallel searching mechanism and the new defined mutation operators, the local searching ability of the algorithm is improved, as well as the algorithm's efficiency.

The numerical results of the simulations verified the advantages of the proposed approach.

Since in the proposed approach any number of objectives can be considered, the authors are currently exploring further extensions of the proposed methodology to include more objective functions or constraints with regard to more realistic problems, as well as other data sets and standard test problems.

\section{REFERENCES}

[1] Lamont, J.W. and Obessis, E.V. 1995."Emission dispatch models and algorithms for the 1990's", IEEE Trans. Power Systems. Vol. 10, No. 2. 941- 947.

[2] Huneault, M. and Galiana, F.D. 1991. "A Survey of the Optimal Power Flow Literature, IEEE Transactions on Power Systems. Vol. 6, No. 2. 762-770. 
[3] Talaq, J.H., El-Hawary, F., El-Hawary, M.E. 1994. "A summary of environmental/ economic dispatch algorithms", IEEE Trans. Power Systems. Vol. 9, No. 3.1508-1516.

[4] Ramanathan, R. 1994. "Emission constrained economic dispatch", IEEE Transactions on Power Systems. 9(4), 1994-2000.

[5] Chang, C.S., Wong, K.P. \& Fan, B. 1995. "SecurityConstrained multiobjective generation dispatch using bicriterion global optimization", IEE Proceedings Generation Transmission and Distribution. 142(4), 406414.

[6] Dhillon, J.S., Parti, S.C. \& Kothari, D.P. 1993. "Stochastic economic emission load dispatch", Electric Power System Resource. 26, 186-197.

[7] Chaaban, F.B., Mezher, T. and Ouwayjan, M. 2004. "Options for emissions reduction from power plants: an economic evaluation", International Journal of Electrical Power \& Energy Systems. Vol. 26, No. 1. 57-63.

[8] Palanichamy, C. and Srikrishna, K. 1991. "Economic thermal power dispatch with emission constraint", Journal of the Institution of Engineers.72, 11-18.

[9] Michalewicz, Z., Logan,T. and Swaminathan, S. 1994. "Evolutionary operators for continuous convex parameter space", Proceedings of Third Annual Conference on Evolutionary Programming. 84-97.

[10] George, E.E. 1943. "Intrasystem transmission lased", AIEE Trans. (Electrical Engineering). vol. 62. 153-158.

[11] Murata T, Ishibuchi H, Tanaka H. 1996. "Multi-objective genetic algorithm and its applications to flowshop scheduling", Computing Eng. 30(4):957-68.
[12] Branke, J. 1999. "Memory enhanced evolutionary algorithms for changing optimization problems", Proc. of the Congress on Evolutionary Computation, vol. 3, 18751882.

[13] Ng, K.P. and Wong, K.C. 1995. "A new diploid scheme and dominance change mechanism for non-stationary function optimization", Proc. of the 6th International Conference on Genetic Algorithms.

[14] Goldberg, D.E. Smith, R. E. 1992. „Diploidy and dominance in artificial genetic search", Complex Systems. $6,21-285$.

[15] Calabretta, R., Galbiati, R., Nolfi, S. and Parisi, D. 1996. "Two is Better than One: a Diploid Genotype for Neural Networks, Neural Processing Letters. 4, 1-7.

[16] Wang, Y., Gao, S. Zhang, H. and Tang, Z. 2010. “An Improved Genetic Algorithm Based Complex-valued Encoding", IJCSNS International Journal of Computer Science and Network Security. VOL.10 (6). 168-174.

[17] Demirel, D., Demirören, A. 2003. "Economic and Minimum Emission Dispatch"”, ELECO. 27-31.

[18] Kulkarni, P.S., Kothari, A.G. and Kothari, D.P. 2000. "Combined economic and emission dispatch using improved backpropagation neural network", Elect. Mach. Power Syst. vol. 28, no.1. 31-44.

[19] Muralidharan, S., Srikrishna, K. and Subramanian, S. 2006. "Emission constrained economic dispatch - A new recursive approach", Electric Power Components and Systems. 34, 343-353.

[20] Balamurugan, R. and Subramanian, S. 2008. "A simplified recursive approach to combined economic emission dispatch", Electric Power Components and Systems. 36, 17- 27. 